\title{
Blocking Mineralocorticoid Receptors Impairs, Blocking Glucocorticoid Receptors Enhances Memory Retrieval in Humans
}

\author{
Ulrike Rimmele*, , Luciana Besedovsky ${ }^{2,3}$, Tanja Lange ${ }^{2,3}$ and Jan Born ${ }^{2,4}$ \\ 'Department of Neurosciences, University of Geneva, Geneva, Switzerland; '2Department of Medical Psychology and Behavioral Neurobiology, \\ University of Tübingen, Tübingen, Germany; ${ }^{3}$ Department of Neuroendocrinology, University of Lübeck, Lübeck, Germany; ${ }^{4}$ Center for Integrative \\ Neuroscience, University of Tübingen, Tübingen, Germany
}

\begin{abstract}
Memory retrieval is impaired at very low as well as very high cortisol levels, but not at intermediate levels. This inverted- $U$-shaped relationship between cortisol levels and memory retrieval may originate from different roles of the mineralocorticoid (MR) and glucocorticoid receptor (GR) that bind cortisol with distinctly different affinity. Here, we examined the role of MRs and GRs in human memory retrieval using specific receptor antagonists. In two double-blind within-subject, cross-over designed studies, young healthy men were asked to retrieve emotional and neutral texts and pictures (learnt 3 days earlier) between 0745 and 0915 hours in the morning, either after administration of $400 \mathrm{mg}$ of the MR blocker spironolactone vs placebo $(200 \mathrm{mg}$ at 2300 hours and $200 \mathrm{mg}$ at 0400 hours, Study I) or after administration of the GR blocker mifepristone vs placebo (200 mg at 2300 hours, Study II). Blockade of MRs impaired free recall of both texts and pictures particularly for emotional material. In contrast, blockade of GRs resulted in better memory retrieval for pictures, with the effect being more pronounced for neutral than emotional materials. These findings indicate indeed opposing roles of MRs and GRs in memory retrieval, with optimal retrieval at intermediate cortisol levels likely mediated by high MR but concurrently low GR activation.

Neuropsychopharmacology (2013) 38, 884-894; doi:I0.1038/npp.2012.254; published online 23 January 2013
\end{abstract}

Keywords: cortisol; spironolactone; mifepristone; mineralocorticoid receptor; glucocorticoid receptor; memory

\section{INTRODUCTION}

Animal and human studies have consistently shown that memory retrieval is impaired under elevated glucocorticoid levels as they occur during stress or after pharmacological manipulation (de Quervain et al, 1998, 2000; Domes et al, 2005; Kuhlmann et al, 2005; Buchanan et al, 2006; Buchanan and Tranel, 2008; Wolf, 2009). Memory retrieval is also significantly impaired when cortisol levels are minimal (Rimmele et al, 2010). In that study, the cortisol synthesis inhibitor metyrapone (3g) almost completely suppressed cortisol levels, resulting in levels during retrieval testing that were distinctly lower than those normally observed during the nadir of endogenous cortisol release. In contrast, a lower dose of metyrapone with correspondingly higher cortisol levels, did not affect retrieval (de Quervain et al, 1998; Marin et al, 2011). In combination, these findings speak for an inverted- $U$-shaped relationship between circulating glucocorticoid levels and memory retrieval

*Correspondence: Dr $\cup$ Rimmele, Department of Neurosciences, University of Geneva, I rue Michel-Servet, 121 I Geneva, Switzerland, Tel: + 4l 22379 5357, Fax: +4l 223795979 ,

E-mail: ulrike.rimmele@unige.ch

Received 5 October 2012; revised 23 November 2012; accepted 26 November 2012; accepted article preview online 6 December 2012
(Reul and de Kloet, 1985; Lupien and Lepage, 2001; Domes et al, 2005; Marin et al, 2011). We supposed that this inverted- $U$-shaped relationship results from differential contributions of the two main corticosteroid receptors.

Glucocorticoids act on two kinds of intracellular receptors, which differ in their affinity and are differentially distributed in the brain. The mineralocorticoid receptors (MRs) bind cortisol with high affinity, resulting in abundant MR occupation even during the circadian nadir of cortisol. In contrast, lower-affinity glucocorticoid receptors (GRs) become increasingly activated only at higher levels of cortisol, when MRs are already occupied to a great extent (Reul and de Kloet, 1985; Joels and de Kloet, 1994; Kalman and Spencer, 2002). Predominant GR activation at high cortisol levels could explain the memory retrieval impairment consistently observed after a stressor or administration of glucocorticoids. In contrast, the retrieval impairment at minimal cortisol levels, resulting from pharmacological blockade of cortisol synthesis, could reflect a consequence of insufficient MR occupation. Optimal memory retrieval is expected when MRs are occupied to a great extent but not GRs (de Kloet et al, 1998; Yau et al, 2011).

In two studies we tested the effects of the MR antagonist spironolactone (Study I) and the GR antagonist mifepristone (Study II) on retrieval of emotional and neutral 
memories in humans. If insufficient occupation of MRs at minimum cortisol concentrations (after high doses of metyrapone) underlies the previously observed retrieval impairment, blockade of MRs should impair memory retrieval. This impairment might be more prominent for emotional than neutral material, as MRs are expressed at high density in the amygdala, a region critically involved in emotional memory (Buchanan, 2007; Yau et al, 2011; Zhou et al, 2011). For GR blockade we hypothesized opposite effects. If high cortisol levels, as they naturally occur under stress or during the morning cortisol rise, saturate GRs and subsequently impair memory retrieval, blockade of GRs should enhance retrieval. Effects mediated by GRs on retrieval could be similar for emotional and neutral material since, in contrast to MRs, GRs are widely distributed throughout the brain regions relevant for memory, including the amygdala, hippocampus and prefrontal cortex (Perlman et al, 2007).

\section{MATERIALS AND METHODS}

\section{Participants}

Sixteen men (mean $\pm S D ; 21.2 \pm 2.29$ years; body mass index (BMI), $22.48 \pm 1.95 \mathrm{~kg} / \mathrm{m}^{2}$ ) participated in Study I. Sixteen other men ( $23.31 \pm 3.64$ years; BMI, $\left.23.47 \pm 1.51 \mathrm{~kg} / \mathrm{m}^{2}\right)$ participated in Study II. The studies were restricted to men due to anticipation of side effects in women, such as early abortion, as they have been previously reported after a dose of $200 \mathrm{mg}$ mifepristone (Li et al, 1988; Swahn et al, 1990; Spitz and Bardin, 1993; Schaff et al, 2000). Participants were non-smokers, on no medication, free of any neurological and psychological disorders, endocrine dysfunction, drug abuse, and reported a normal sleep-wake cycle. Participants acclimated to sleeping in the laboratory by spending one night in the laboratory. On experimental days, participants were asked to get up before 0700 hours, not take any naps during the day, and abstain from alcohol or (after 1200 hours) caffeine. The study was approved by the local ethics committee. Subjects gave written informed consent and were paid for participation.

\section{Experimental Design and Procedure}

Each of the studies employed a randomized, placebocontrolled, double-blind, within-subject cross-over design. Each participant was tested in two conditions (active agent $v s$ placebo) with the order of conditions balanced across subjects. The two conditions for a subject were separated by an interval of at least 12 days.

Each condition included a learning session and a retrieval session (Figure 1a). In the learning session (between 0800 and 1100 hours) participants memorized emotional and neutral texts and pictures. Three days later, participants recalled the texts and pictures between 0745 and 0915 hours following a night in the sleep laboratory (lights off at 2300 hours) during which two oral doses of spironolactone (200 mg each, UK-SH Pharmacy, Lübeck, Germany; half-life in plasma $1-2 \mathrm{~h}$, half-life in plasma of the active metabolite canrenone $18-23 \mathrm{~h}$ ) or placebo were administered at 2300 and 0400 hours. In Study II, instead of spironolactone, a dose of $200 \mathrm{mg}$ of the glucocorticoid antagonist mifepristone
(Exelgyn Laboratories, Paris, France; half-life in plasma $>24 \mathrm{~h}$ ) was administered orally at 2300 hours. To keep the procedures consistent across studies, participants were also woken up at 0400 hours, but administered placebo.

Retrieval was tested during the morning hours, because owing to the spontaneous morning rise in cortisol, this time interval was expected to be particularly sensitive to effects of blocking corticosteroid receptors. The doses and timing of spironolactone and mifepristone administration were established based on pilot experiments indicating efficacy of this schedule of administration as assessed by pituitaryadrenal responses, that is, changes in plasma adrenocorticotropine (ACTH) and cortisol (Dodt et al, 1993; Born et al, 1997; Wellhoener et al, 2004).

\section{Memory Tests}

Texts. To assess the influence of medication $v s$ placebo on memory retrieval, two parallel versions of emotional and neutral texts were used (Wagner et al, 2001, 2005; Groch et al, 2011; Wilhelm et al, 2011).

In the learning session, the subjects learned one emotional and one neutral text, embraced by two additional neutral texts that served as primacy and recency buffers. The order of the experimental texts was balanced within a session and across the subject's two test occasions. Participants were instructed to read and memorize the texts in detail within $4 \mathrm{~min}$. They were informed that recall would be tested immediately after learning as well as later at the retrieval session. Using the Self-Assessment Manikin (SAM) scale (1, highly positive; 9 , highly negative; 1 , very much arousing; 9, not at all arousing), participants in both studies rated the emotional texts with greater negative valence (emotional, $6.42 \pm 0.21$; neutral, $4.63 \pm 0.18$; $\left.t_{(31)}=7.98 ; p<0.001\right)$ and higher arousal (emotional, $4.31 \pm 0.27$; neutral, $\left.6.87 \pm 0.23 ; t_{(31)}=7.83 ; p<0.001\right)$. After learning, subjects were asked to write down the texts as exactly as possible with no time limit using separate sheets of paper for each text. No restriction was given concerning the order of text retrieval. This free recall test served to determine how much information was initially encoded, thereby providing an individual baseline value. Recall was tested in the same way in the retrieval session 3 days later, after administration of the active agents or placebo.

Memory performance was based on the number of correctly recalled content words. Validity of this measure was confirmed in previous experiments (Schuerer-Necker, 1994). Two independent experimenters, blind to the drug condition, assessed the number of recalled content words for each story per participant ( $r=0.93$ to $r=0.99$, for both studies). Retrieval performance was determined by the difference in the number of content words recalled during the retrieval session minus the number of content words recalled immediately after learning (baseline). Note this is a measure of 'forgetting', with lower negative values indicating better retention. Additionally, the absolute number of recalled content words was examined.

At the retrieval session, after free recall, a recognition memory test of the texts was performed (Swain et al, 1998; Krug et al, 2006). For each story, 12 word pairs were presented with one word representing a content word of the story and the other one a synonym. To test recognition, 

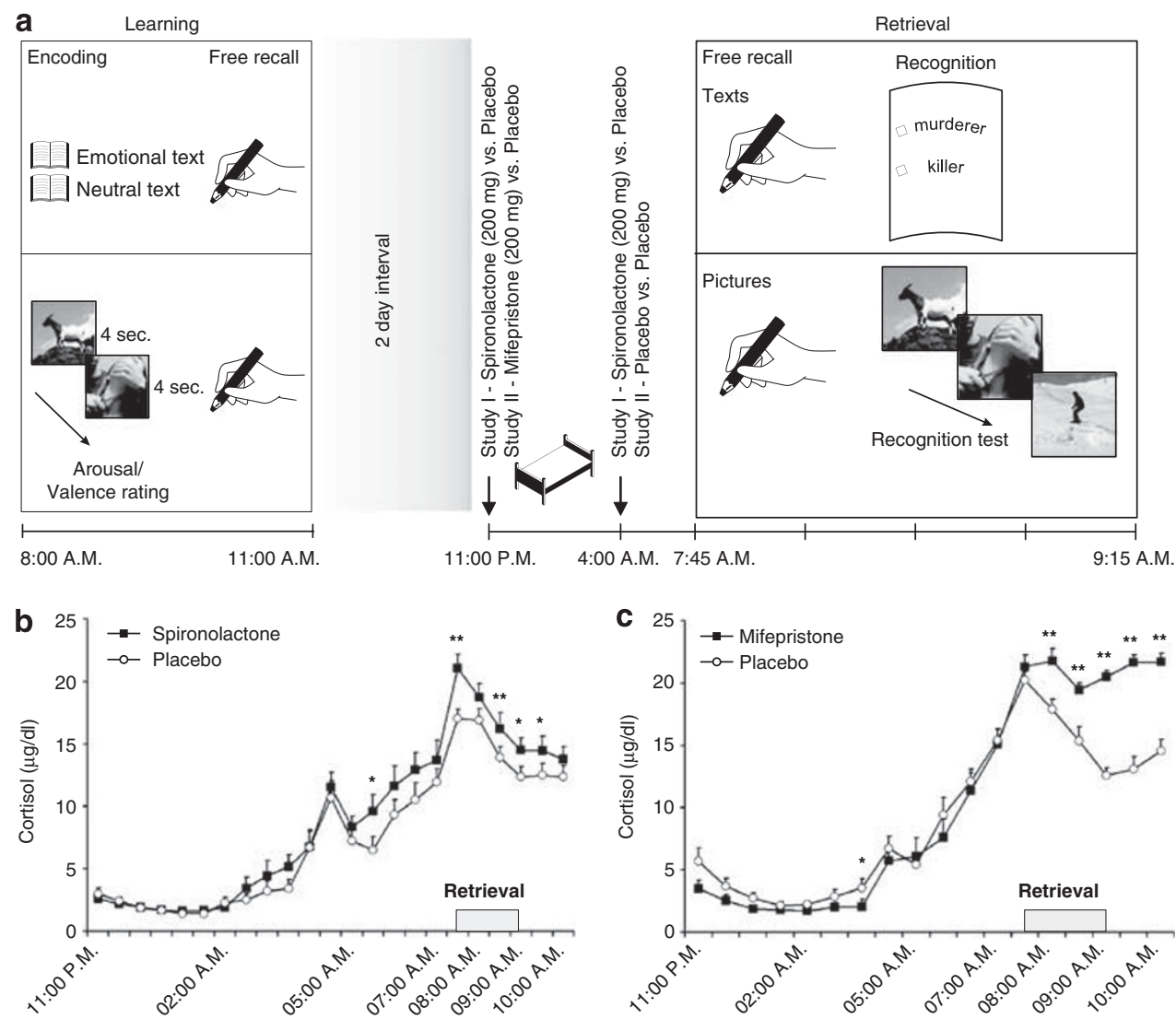

Figure I (a) Experimental procedure. At Learning, participants learned and immediately recalled texts and pictures of emotional and neutral nature. Three days later, retrieval of the materials (Free recall followed by Recognition tests) was tested between 0745 and 0915 hours, that is, the time of the cortisol morning rise. Participants spent the night before retrieval testing in the sleep laboratory and were administered placebo or the active agent. In Study I, the active agent was the MR blocker spironolactone (200 mg, at 2300 hours and $200 \mathrm{mg}$ at 0400 hours). In Study II, participants received $200 \mathrm{mg}$ of the GR blocker mifepristone at 2300 hours. To keep procedures consistent across both studies, in Study II participants were also woken up at 0400 hours but received placebo. At least 10 days later, the procedure was repeated with different material and participants received the treatment they had not received the first time. (b) Mean \pm SEM plasma cortisol concentration at retrieval. Horizontal bar indicates time of testing. Spironolactone (filled squares) and (c) mifepristone (filled squares) led to a stronger increase in the morning cortisol rise compared with the placebo condition (empty circles). $* * *<0.01$; * $p<0.05$ for pairwise comparisons between the treatment conditions at the different time points. The displayed images contain similar content as the IAPS images that were used in the studies.

subjects identified for each word pair, which one of the two words had occurred in the text.

Pictures. Three hundred pictures from the International Affective Picture System (Lang et al, 2005) were divided into two parallel versions ( 75 negative, 75 neutral per version) that were counterbalanced across active agent $v s$ placebo conditions. In both studies, participants rated the emotional and neutral pictures different in terms of valence (mean \pm SEM; emotional, $6.16 \pm 0.16$; neutral, $4.37 \pm 0.12 ; t_{(31)}=$ $11.40 ; p<0.001$ ) and arousal (emotional, $4.72 \pm 0.22$; neutral, $\left.6.52 \pm 0.22 ; t_{(31)}=9.55 ; p<0.001\right)$ using the SAM (Bradley et al, 1992).

In the learning session, participant's were instructed to memorize as much as possible of the 100 pictures (50 neutral, 50 negative, each presented for $4 \mathrm{~s}$ ) and rate them on valence and arousal. Immediately after learning, participants freely recalled the pictures by writing down a detailed description of every picture that they could remember. No restriction was given concerning the order of picture retrieval. This test determined the initial encoding level of both the negative and neutral pictures, as an individual baseline for delayed retrieval performance (by a difference score 'delayed retrieval minus immediate recall after learning'). Two independent experimenters, blind to the drug condition, assessed the number and details of recalled pictures per participant $(r=0.80$ to $r=0.98$, for both studies).

In the retrieval session, after the administration of active agents or placebo, the same free recall procedure was used. Then recognition memory was tested for the 100 studied pictures vs 50 novel pictures (25 neutral, 25 negative). Subjects indicated whether they had seen the picture at learning or whether it was new.

\section{Psychological Control Variables}

At the beginning of the learning and retrieval sessions, attention, positive and negative affect, and working memory were assessed using the $\mathrm{d} 2$ letter cancellation test (Brickenkamp and Zillmer, 1998), the Positive and Negative Affect Scale (Watson et al, 1988), and the Digit Span subtest (forward, backward) of the Wechsler Adult Intelligence Scale (Wechsler, 1981). Additionally at the end of the retrieval session, working memory was assessed with the 
Sternberg task (Sternberg, 1966) as described in Lupien et al (1999) with 20 trials per comparison load. For each comparison load, the target was present in 10 trials and absent in another 10 trials.

\section{Hormones and Sleep}

To assess the effects of medication $v s$ placebo on cortisol, ACTH, epinephrine, norepinephrine levels, in Study I, blood samples were collected every 30 min from 2300 hours until 1000 hours via a long plastic tube from an adjacent room. In Study II, blood samples were collected every $45 \mathrm{~min}$ from 2300 hours until 0630 hours, and every 30 min from 0630 hours until 1000 hours. Blood samples were immediately centrifuged and then stored at $-80^{\circ} \mathrm{C}$ until assay. Serum cortisol concentrations were assessed using the Immulite (Siemens Medical Solutions Diagnostics, Los Angeles, CA; serum sensitivity, $0.2 \mu \mathrm{g} / \mathrm{dl}$, interassay coefficient of variation $<10 \%)$. ACTH was assessed in plasma (Immulite, Siemens Medical Solutions Diagnostics, Los Angeles, CA; sensitivity, $9 \mathrm{pg} / \mathrm{ml}$, interassay coefficient of variation $<9.6 \%$ ). Plasma epinephrine (E) and norepinephrine (NE) were assessed with standard high-performance liquid chromatography (ChromSystems, Munich, Germany; E sensitivity, $2 \mathrm{pg} / \mathrm{ml}$; interassay coefficient of variation $<6.5 \%$; NE sensitivity, $5 \mathrm{pg} / \mathrm{ml}$; interassay coefficient of variation $<6 \%$ ). All samples from an individual were determined in the same assay. Because $\mathrm{E}$ levels during the night were mostly below the detection threshold they are not reported here.

Sleep was assessed by standard polysomnographical recordings scored offline according to the criteria by Rechtschaffen and Kales (1968). Subjective sleep quality was assessed by the SFA questionnaire ('Schlaffragebogen-A') immediately after wakening (Görtelmeyer, 1985).

\section{Statistical Analyses}

Statistical analysis was based on analyses of variance (ANOVA) with repeated-measures factors for the treatment condition (active agent vs placebo) and, for memory variables, with the additional factor 'emotionality' (neutral $v s$ emotional). For psychological variables (attention, affect, working memory), ANOVA included an additional repeated-measures factor 'learn/retrieval'. For hormone levels, analyses included repeated-measures factor 'time of measurements'. Where appropriate, Greenhouse-Geisser corrections of degrees of freedom were used. Significant ANOVA effects were specified by $t$ tests. Additionally Cohen's d effect sizes were calculated.

\section{RESULTS-BLOCKING MR (STUDY I)}

\section{Memory for Texts}

Retrieval session. In the retrieval session, when given spironolactone, participants recalled distinctly less of the texts (Table 1). The difference score between recalled content words at the retrieval minus immediate recall after learning indicated significantly greater forgetting, that is, diminished recall after spironolactone than placebo $\left(\mathrm{F}_{(1,15)}=5.64 ; p<0.05\right.$, for treatment main effect). Recall of emotional texts suffered most under spironolactone ( $-9.56 \pm 1.48$; placebo, $-3.34 \pm 1.58$ for difference scores; $t_{(15)}=2.55 ; p<0.05 ; d=1.02 ; \mathrm{F}_{(1,15)}=4.98 ; p<0.05$ for treatment $\times$ emotionality). For neutral texts, a difference in the same direction was not significant (spironolactone, $-5.00 \pm 1.08 ;$ placebo, $-3.65 \pm 0.74 ; d=0.36 ; p>0.28$, Figure 2). The pronounced retrieval impairment of emotional texts after spironolactone was also evident in the absolute number of recalled content words (spironolactone, $32.53 \pm 3.17$; placebo, $38.37 \pm 2.14 ; \mathrm{F}_{(1,15)}=7.98 ; p<0.05$ for treatment $\times$ emotionality). Recognition of content words measured after free recall did not differ between the treatment conditions (all $p>0.70$ ).

Learning session. In the learning session (before treatment) immediate recall, an estimate of encoding did not differ between the spironolactone and placebo condition $(p>0.16$ for treatment main effect and treatment $x$ emotionality interaction). Independent of the condition, participants recalled more content words of emotional than neutral texts in the learning and retrieval session $(p<0.001)$.

\section{Memory for Pictures}

Retrieval session. Similar to its impairing effect on memory retrieval for texts, participants also recalled less pictures under spironolactone than under placebo (for difference scores compared with performance at learning combined for emotional and neutral pictures: $-12.5 \pm 0.72 v s-8.13 \pm 1.40 ; \mathrm{F}_{(1,15)}=6.57 ; p<0.05$ for treatment main effect, Table 1). This decrease was most pronounced for retrieval of emotional pictures (spironolactone, $-7.91 \pm 0.64$; placebo, $-4.03 \pm 0.72, t_{(15)}=3.33$; $p<0.01 ; d=1.42)$. For neutral pictures, a difference in the same direction remained non-significant (spironolactone, $-4.63 \pm 0.50 ; \quad$ placebo $-4.09 \pm 0.76 ; \quad p>0.52 ; \quad d=0.21$; $\mathrm{F}_{(1,15)}=10.57 ; \quad p<0.01, \quad$ for treatment $\times$ emotionality, Figure 2). In parallel, the marked retrieval impairment after spironolactone tended to be evident in the absolute number of recalled pictures $\left(\mathrm{F}_{(1,15)} 3.85 ; p=0.06\right.$ for treatment main effect; $\left.t_{(15)}=1.96 ; p=0.06\right)$, in particular for the number of emotional pictures (spironolactone, $16.78 \pm 1.01$; placebo, $\left.19.75 \pm 1.55, t_{(15)}=2.17 ; p<0.05 ; d=0.57\right)$.

Retrieval of picture details was also impaired after spironolactone (Table 1). Participants tended to recall less details under spironolactone than placebo (for difference scores combined for emotional and neutral picture details in comparison with performance at learning $-29.62 \pm 2.91$ $v s-21.90 \pm 3.55 ; \mathrm{F}_{(1,15)}=2.98 ; p=0.10$ for treatment main effect). The effect was particularly consistent for recalled details of emotional pictures (spironolactone, $-18.41 \pm$ 1.79; placebo, $-11.43 \pm 1.70, t_{(15)}=2.73 ; p<0.05 ; d=1.00$, $\mathrm{F}_{(1,15)}=6.62 ; p<0.05$ for treatment $\times$ emotionality), whereas difference scores for recalled details of neutral pictures did not differ (spironolactone, $-11.22 \pm 1.54$; placebo, $-10.47 \pm 2.11 ; \quad t_{(15)}=0.30 ; p>0.76 ; d=0.10$, Figure 2$)$. Similarly, for the absolute number of recalled details, the retrieval impairment under spironolactone was more consistent for emotional (25.78 \pm 1.75 ; placebo, $32.88 \pm$ $\left.2.69 ; t_{(15)}=2.52 ; p<0.05 ; d=0.78\right)$ than neutral pictures (spironolactone, $12.57 \pm 1.19$; placebo, $15.22 \pm 1.68 ; p>0.14$; 
Table I Memory Retrieval Under Spironolactone and Placebo

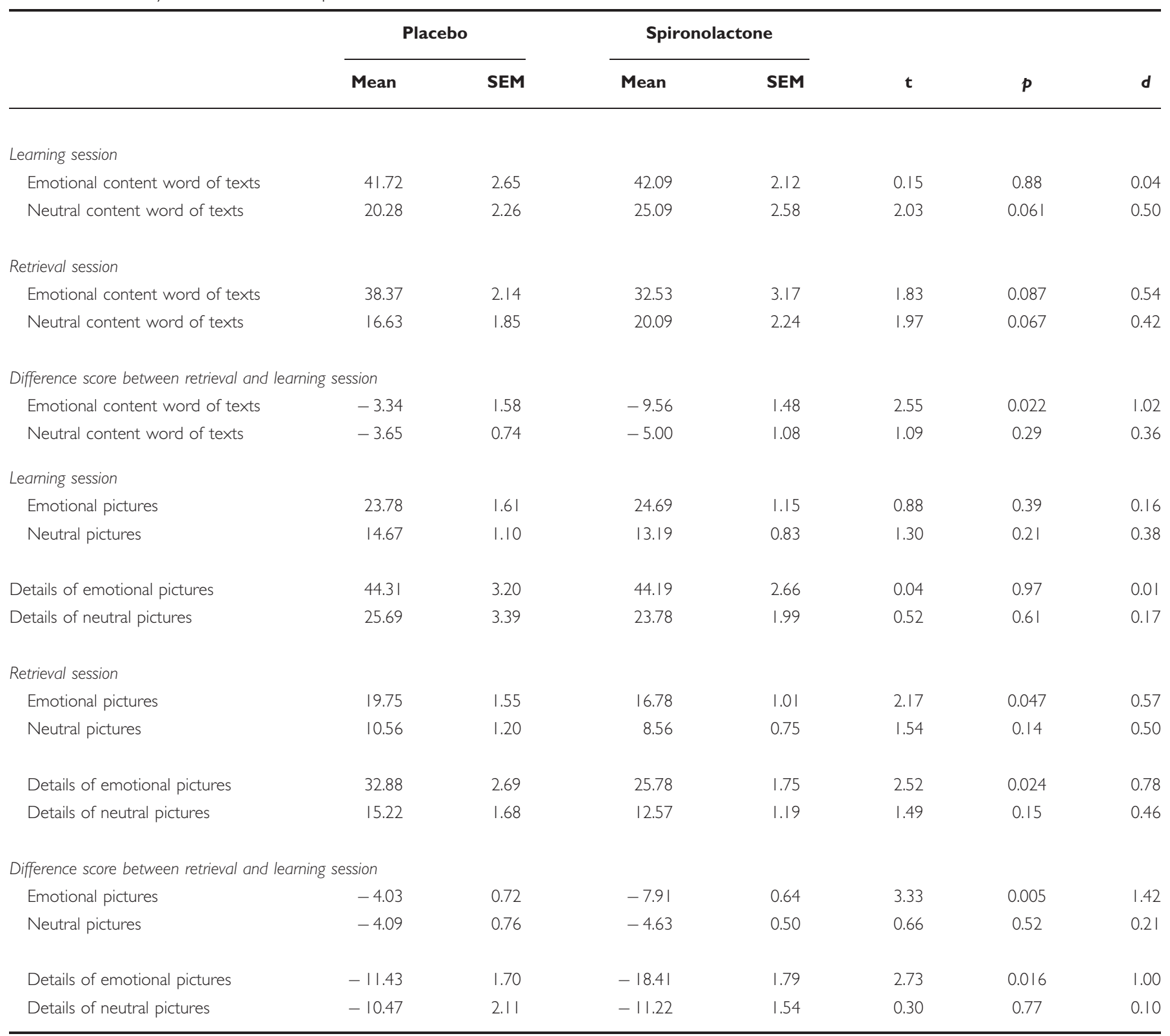

$t=t$ value; $p=p$ value; $d=$ effect size (Cohen's $d$ ). The medication was only administered at the retrieval session. Retrieval at the learning session served to determine individual baseline levels of memory performance.

$d=0.46, \mathrm{~F}_{(1,15)}=5.19 ; p<0.05$ for treatment main effect; $\mathrm{F}_{(1,15)}=5.02 ; p<0.05$ for treatment $\times$ emotionality). Recognition of pictures tested after free recall, was not affected by spironolactone.

Learning session. In the learning session (before treatment), participants recalled similar amounts of pictures as well as picture details in the spironolactone and placebo condition (values of $p>0.20$, Table 1 ). Generally, participants recalled more emotional than neutral pictures and more details thereof $(p<0.001$ for emotionality main effects).

\section{Hormones, Sleep and Control Variables}

Plasma cortisol levels after spironolactone increased after 0400 hours compared with the placebo condition $\left(\mathrm{F}_{(1,15)}=13.75 ; p<0.01\right.$ for treatment main effect in an analysis on the interval between 0430 and 1000 hours) while before this interval cortisol levels did not differ (values of $p>0.33$, Figure 1b). Peak concentrations of cortisol occurred during the time of retrieval testing (between 0745 and 0915 hours) and, here, were on average higher in the spironolactone $(17.01 \pm 0.95 \mu \mathrm{g} / \mathrm{dl})$ than placebo condition $\quad\left(14.53 \pm 0.67 \mu \mathrm{g} / \mathrm{dl} ; \quad t_{(15)}=3.44 ; \quad p<0.01\right.$, Figure 1b). Plasma ACTH and NE levels did not differ between treatment conditions $(p>0.25)$. Independent of the 

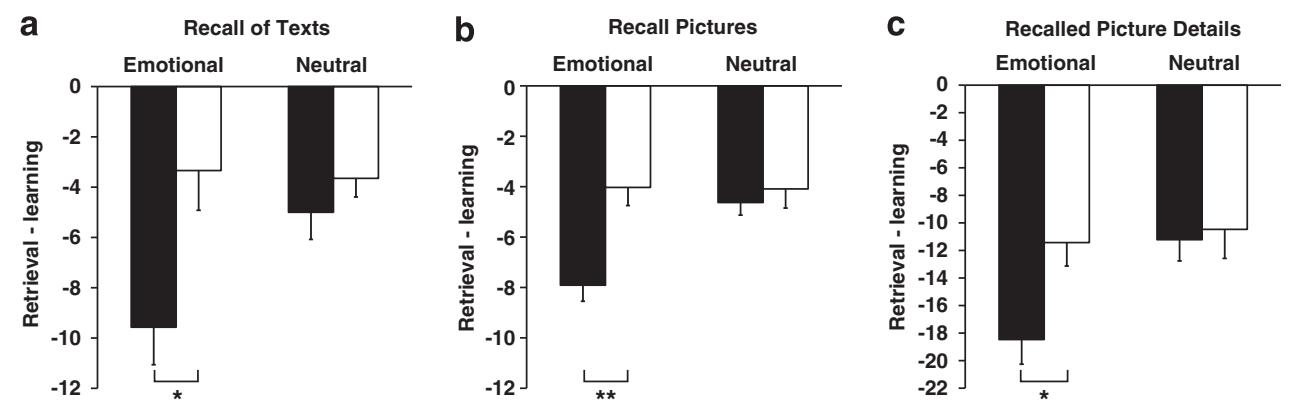

Figure 2 Effects of spironolactone (MR blocker) on memory retrieval. Free recall performance is indicated by difference scores between the number of recalled words, pictures or picture details at the retrieval session minus immediate recall performance at learning, serving as baseline encoding measure. Note, negative values of this measure reflect forgetting across the retention interval with smaller negative values indicating better retrieval performance. Compared with placebo (empty bars), the MR antagonist spironolactone (black bars) reduced (a) free recall of emotional texts, and (b) free recall of emotional pictures, and (c) of details from emotional pictures. Mean values \pm SEM are indicated. $* * p<0.0$ I; $* p<0.05$ for pairwise comparisons between the treatment conditions.

treatment, levels of the three hormones exhibited the expected increase towards the end of the night $(p<0.001$ for main effects of time).

Spironolactone did not significantly affect any sleep parameter $(p>0.17)$ or self-reported sleep quality for the total night $(p>0.60)$. Also, attention performance ( $\mathrm{d} 2$ test) did not differ between the treatments in the learning or retrieval session in the raw measures of hits, false alarms and misses $(p>0.26)$ as well as for 'hits minus false alarms' (spironolactone, $559.19 \pm 16.69$; placebo, $573.62 \pm 16.28$; $p>0.35$ ). Likewise, there were no differences between treatment conditions in performance on the Digit Span test $(p>0.41)$, the Sternberg task $(p>0.13)$, and positive and negative affect $(p>0.15)$ in both the learning and the retrieval session. When asked at the end of the retrieval sessions, participants were not able to correctly identify whether they had received an active agent or placebo $\left(\chi^{2}\right.$ test; $\left.p>0.65\right)$. No side effects of spironolactone were observed.

\section{RESULTS-BLOCKING GR (STUDY II)}

\section{Memory for Texts}

Retrieval session. In the retrieval session, after mifepristone compared with placebo, participants demonstrated neither a change in the difference scores (ie, the number of recalled content words at retrieval minus immediate recall performance after learning), nor in the absolute number of recalled content words $(p>0.50$, Table 2$)$, nor in recognition $(p>0.64)$.

Learning session. At the learning session (before treatment), recall did not differ between conditions $(p>0.43$, Table 2), but was generally higher for emotional than neutral texts $(p<0.001)$.

\section{Memory for Pictures}

Retrieval session. In the retrieval session, mifepristone distinctly improved recall of neutral pictures (for difference scores between retrieval performance minus immediate recall at learning; mifepristone, $-2.81 \pm 0.48$; placebo, $\left.-4.59 \pm 0.83 ; t_{(15)}=2.11 ; p<0.05 ; d=0.66\right)$, but not of emotional pictures $(-5.97 \pm 0.78$; placebo, $-5.47 \pm 0.83$, $p>0.61 ; d=0.15 ; \mathrm{F}_{(1,15)}=4.54 ; p<0.05$ for treatment $\times$ emotionality, Figure 3$)$. This retrieval improvement was not evident for absolute number of pictures recalled $(p>0.56)$, possibly due to the fact that immediate recall of the number of neutral pictures at the learning session was slightly better in the placebo $(11.78 \pm 1.23)$ than mifepristone condition $\left(9.50 \pm 0.81 ; t_{(15)}=2.28 ; p<0.05 ; d=0.55\right.$, Table 2$)$.

There was a very prominent retrieval enhancement after mifepristone for the measure of recalled picture details (Figure 3). Under mifepristone participants not only showed higher retention of details of neutral pictures (mifepristone, $-5.75 \pm 1.55$; placebo $-9.88 \pm 1.63$; $\left.t_{(15)}=2.97 ; p=0.01 ; d=0.65\right)$, but also of emotional pictures (difference scores; mifepristone, $-11.56 \pm 1.55$; placebo, $\quad-16.16 \pm 1.77 ; \quad t_{(15)}=2.59 ; \quad p<0.05 ; \quad d=0.69$; $\mathrm{F}_{(1,15)}=19.92 ; p<0.001$ for treatment main effect). Recognition of pictures was not affected by mifepristone. Independent of the treatment, participants recalled more emotional than neutral pictures $(p<0.001)$.

Learning session. In the learning session (before treatment), participants recalled similar amounts of emotional pictures as well as details of both emotional and neutral pictures in the mifepristone and placebo condition (values of $p>0.27$, Table 2). However, the number of neutral pictures at learning was slightly better in the placebo $(11.78 \pm 1.23)$ than mifepristone condition $(9.50 \pm 0.81$; $\left.t_{(15)}=2.28 ; \quad p<0.05 ; \quad d=0.55\right)$. Generally, participants recalled more emotional than neutral pictures and more details thereof ( $p<0.001$ for emotionality main effects).

\section{Hormones, Sleep and Control Variables}

Plasma cortisol levels increased more throughout the night, that is, from 2300 hours to 1000 hours under mifepristone than placebo $\left(\mathrm{F}_{(17,255)}=14.81 ; p<0.001\right.$ for treatment $\times$ time, Figure 1c). Importantly, peak concentrations of cortisol occurring during the time of retrieval testing (between 0745 and 0915 hours), were higher in the mifepristone $(20.94 \pm 0.53 \mu \mathrm{g} / \mathrm{dl})$ than placebo condition $\left(15.85 \pm 0.55 \mu \mathrm{g} / \mathrm{dl} ; t_{(15)}=8.71 ; p<0.01\right)$. Plasma ACTH 
Table 2 Memory Retrieval Under Mifepristone and Placebo

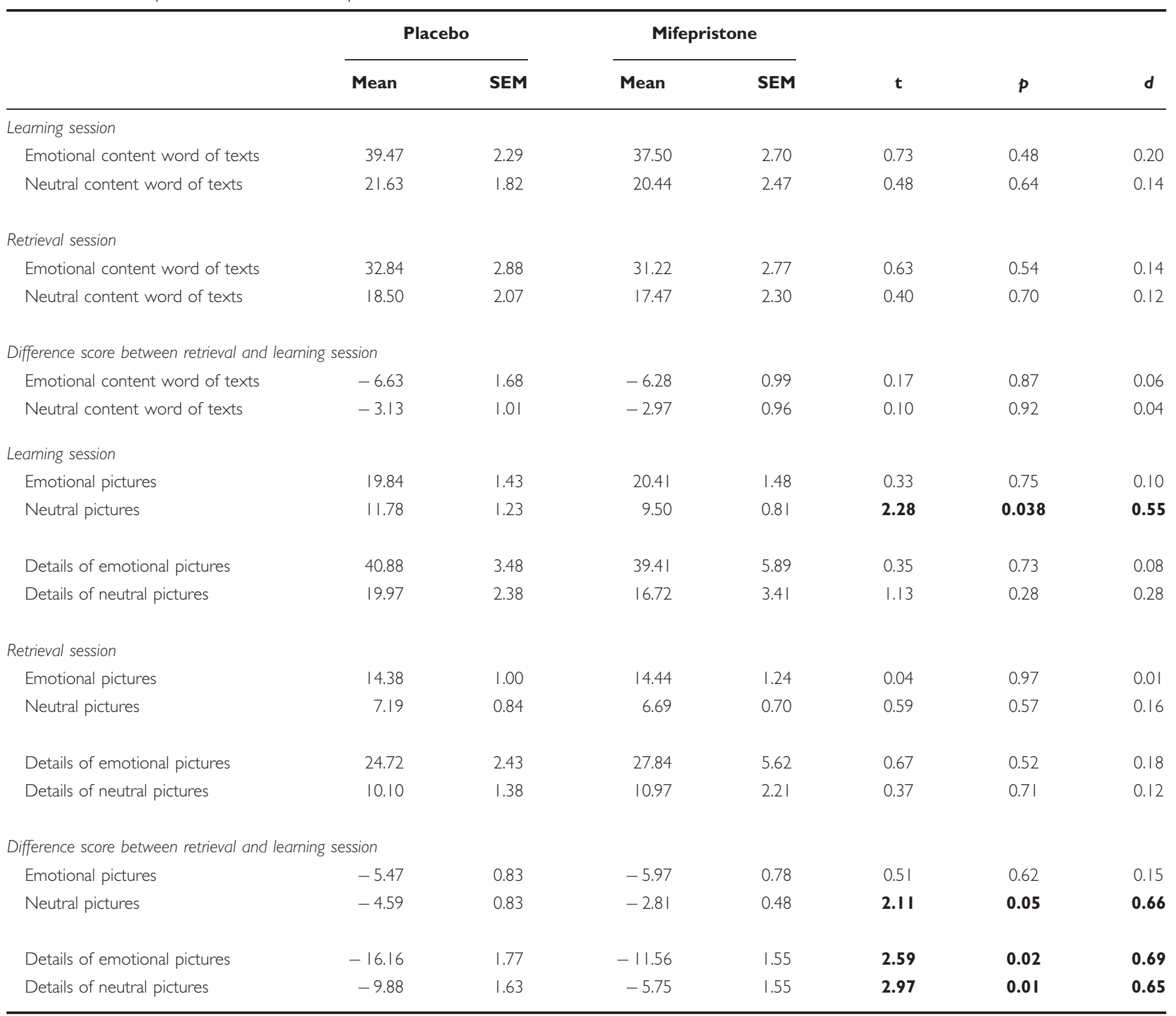

$t=t$ value; $p=p$ value; $d=$ effect size (Cohen's $d$ ). Significant results are displayed in bold. The medication was only administered at the retrieval session. Retrieval at the learning session served to determine individual baseline levels of memory performance.

levels also increased more throughout the night after mifepristone than placebo $\left(\mathrm{F}_{(17,255)}=9.23 ; p<0.001\right.$ for treatment $\times$ time interaction). Again, maximum levels of ACTH occurred during the time of retrieval testing and, here, were on average higher in the mifepristone $(46.13 \pm 2.52 \mathrm{pg} / \mathrm{ml})$ than placebo condition $(30.73 \pm 1.75 \mathrm{pg} /$ $\left.\mathrm{ml} ; t_{(15)}=6.03 ; p<0.001\right)$. Plasma NE levels did not differ between treatments $(p>0.11)$.

Sleep architecture was overall comparable between treatment conditions (values of $p>0.17$ ), except that following mifepristone time awake was slightly increased $\left(57.93 \pm 13.28 \mathrm{~min}\right.$; placebo, $38.97 \pm 12.72 \mathrm{~min} ; t_{(14)}=1.82$; $p=0.091)$ and subjects spent less time in REM sleep $\left(56.13 \pm 5.33 \mathrm{~min} ;\right.$ placebo, $68.33 \pm 4.99 \mathrm{~min} ; \quad t_{(14)}=2.50$ $p<0.05)$. Self-reported sleep quality for the total night did not differ between the treatment conditions $(p>0.43)$.

Attention performance (d2 test) did not differ between the treatment conditions at learning or retrieval in the raw measures of hits, false alarms and misses (values of $p>0.16$ ) and in 'hits minus false alarms' (mifepristone, $534.88 \pm 15.53$; placebo, $520.19 \pm 13.99$; $p>0.25$ ). Likewise, there were no differences between treatment conditions in performance on the Digit Span test $(p>0.16)$, Sternberg task $(p>0.66)$, or in positive and negative affect $(p>0.07)$ in both the learning and the retrieval session. Subjects were not able to correctly identify whether they had received an active agent or placebo ( $\chi^{2}$ test; $\left.p>0.64\right)$. No side effects of mifepristone were observed. 
a

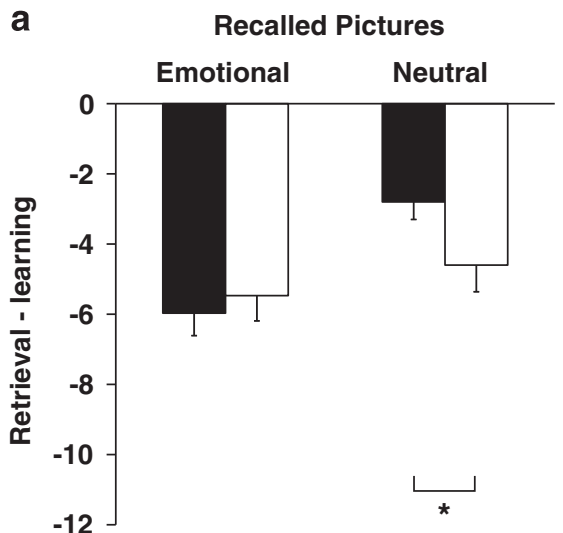

b Recalled Picture Details

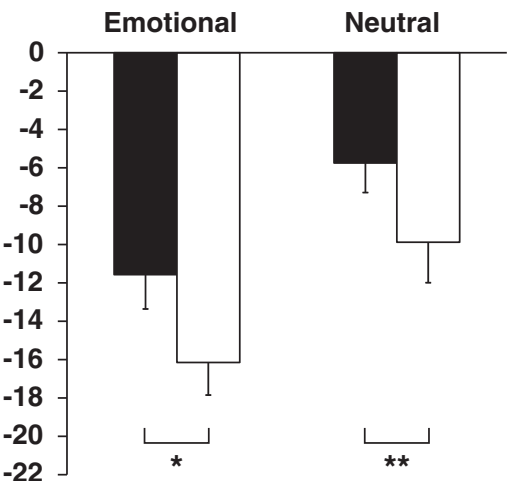

Figure 3 Compared with placebo (empty bars), the GR antagonist mifepristone (black bars) improved (a) free recall of neutral pictures as well as (b) free recall of emotional and neutral picture details. Free recall performance is indicated by difference scores between the number of recalled pictures or picture details at the retrieval session minus immediate recall performance at the learning session. Mean values \pm SEM are indicated. $* * p<0.01$; $* p<0.05$ for pairwise comparisons between the treatment conditions.

\section{DISCUSSION}

In two experiments, we examined the effects of MR or GR antagonists on human memory retrieval. MR blockade led to impairment in free recall, especially of emotional texts and pictures. In contrast, GR blockade resulted in improved free recall of both neutral and emotional pictures, in particular of picture details. These data point towards opposing roles MRs and GRs play in memory retrieval: MRs are crucially involved in effective memory retrieval, whereas activation of GRs may decrease retrieval performance.

Consistent with previous studies in healthy participants and patients with depression (Dodt et al, 1993; Deuschle et al, 1998; Young et al, 1998; Heuser et al, 2000; Otte et al, $2007,2010)$ the MR antagonist spironolactone increased basal cortisol levels, but did not affect ACTH levels. Similarly, consistent with previous findings (Wiedemann et al, 1998), the GR antagonist mifepristone increased morning rises in plasma cortisol and $\mathrm{ACTH}$, resulting in higher concentrations of these hormones during the time of retrieval testing. In contrast, neither spironolactone nor mifepristone affected norepinephrine levels. The disinhibition of the hypothalamus-pituitary-adrenocortical (HPA) axis under both treatments does not only prove the efficacy of the present treatment regimens, but also further confirms the involvement of both MRs and GRs in inhibitory feedback regulation of the HPA system.

The finding that MR blockade impaired free recall of texts and pictures, especially when emotional, is in line with the results of animal studies that found memory retrieval to be impaired after acute MR blockade (Oitzl and de Kloet, 1992; Schwabe et al, 2010; Yau et al, 2011; Zhou et al, 2011) or repeated MR antagonist administration (Douma et al, 1998; Yau et al, 1999). In contrast, blockade of GRs in the present study enhanced free recall of pictures, with an equivalent effect for neutral and emotional picture details. This finding is consistent with animal studies that report GR blockade to abolish the memory retrieval impairment in animals with high hippocampal corticosterone levels (Yau et al, 2011; Dorey et al, 2012). Also, another rodent study suggested an enhancing effect after continuous administration of a GR blocker on retrieval of spatial memory (Oitzl et al, 1998). In line with this finding, a treatment with mifepristone over several days enhanced spatial working memory performance in humans with major depression, apart from benefiting mood in these patients (Belanoff et al, 2001; Young et al, 2004; Watson et al, 2012). However, these studies do not allow the dissociation of effects on retrieval from effects on encoding or consolidation, as the GR blocker was effective during both learning and retrieval. In the present study, the MR and GR antagonist were administered specifically prior to retrieval testing, which took place 3 days after learning. Learning and subsequent consolidation likely terminated during this 3-day interval, excluding any effects of the MR and GR blocker on these memory stages.

We controlled for various factors that could nonspecifically affect memory retrieval. MR blockade did not influence affect, attention and working memory, excluding these factors as confounding variables. Two previous studies reported attention to be impaired after blocking MRs with spironolactone, which might be explained by a different timing and dosage of the MR blocker (Otte et al, 2007; Cornelisse et al, 2011). GR blockade likewise did not influence affect, attention or working memory. Sleep prior to retrieval testing was also comparable between the placebo and active agent conditions in both studies except that on the mifepristone condition subjects were longer awake and showed reduced time in REM sleep, replicating previous findings (Wiedemann et al, 1992, 1998). However, the size of the change was small and sleep disturbing effects like increased time awake or decreased REM sleep are expected to diminish rather than enhance executive functions like retrieval (Durmer and Dinges, 2005; Killgore, 2010) making it highly unlikely that these changes in sleep contributed to the improved retrieval performance during GR blockade.

Basically, the retrieval deficit after MR blockade could either be due to inactive MRs or predominant GR activation. Regarding the latter, it could be argued that increased cortisol levels present during MR blockade added to the retrieval impairment. High cortisol levels following a stressor or pharmacological manipulation, and 
consequently increasingly activated GRs, have indeed been associated with impaired memory retrieval (de Quervain et al, 1998, 2000; Kuhlmann et al, 2005; Dorey et al, 2011). However, compared with previous studies reporting cortisol-associated changes in retrieval, the increase in cortisol levels observed after spironolactone here, although statistically significant, was only of marginal size (averaging $2.48 \mu \mathrm{g} / \mathrm{dl}$ ), which is not expected to become effective at the behavioral level. Nevertheless, considering the presence of substantial cortisol signaling during retrieval, it cannot be ruled out that the retrieval impairment after spironolactone originated primarily from an imbalance in MR/GR activation towards prevailing GR activity, rather than from an immediate lack of MR activity (de Kloet et al, 2000). However, prevailing GR activity alone would not sufficiently explain the retrieval deficit, as it appears to be at odds with the retrieval deficit that has been observed at very low cortisol levels, that is, a condition where GR activation is not prevailing, but actually largely lacking (Lupien et al, 2002; Rimmele et al, 2010; Marin et al, 2011).

Indeed, it appears most straightforward to consider the impaired retrieval an immediate consequence of diminished MR activation due to spironolactone blocking MRs. A primary role for MRs in mediating the retrieval deficits after spironolactone is also consistent with two further findings of this study: (i) the failure of spironolactone to affect recognition and (ii) the greater spironolactone-induced retrieval impairment for emotional as opposed to neutral material. Free recall and recognition involve different brain systems. Free recall critically relies on a fine-tuned interaction between prefrontal and hippocampal circuitry and, therefore is probably most sensitive to disturbing influences mediated via MRs which are expressed at high density in the amygdala and hippocampus (Patel et al, 2000; Pryce, 2008), whereas hippocampal contributions to recognition performance are less essential (Mayes et al, 2002; Holdstock et al, 2005). Second and along the same line, the stronger retrieval impairment for emotional texts and pictures is consistent with the fact that retrieval of emotional memories relies critically on the amygdala (Buchanan, 2007), a region rich of MRs (Patel et al, 2000; Pryce, 2008).

It could be also MRs mediating the retrieval enhancement after GR blockade. GR blockade not only leads to lower activation of GRs, but also to more prominent effects of the high-affinity MR. Comparable results of a previous animal study suggest that simultaneously decreasing GR activation and increasing MR activation by GR antagonism ameliorates spatial memory retrieval impairments (Yau et al, 2011). In addition, several studies in mice that assessed the effects of transgenic overexpression of MR in the forebrain and hippocampus, shifting the balance towards predominant MR activation, demonstrated that chronic elevation of MRs enhances memory (Lai et al, 2007; Ferguson and Sapolsky, 2008).

Of note, the most prominent effect of GR blockade was enhanced memory retrieval for details of pictures. Indeed, one would expect this parameter to be the most sensitive for a retrieval enhancement since it is the most difficult one of the administered memory tests and thus allows most potential for a memory enhancement. To illustrate this, in the placebo condition, subjects at retrieval testing recalled generally distinctly less picture details $(53.7 \pm 3.42 \%$ with reference to learning) than content words $(83.64 \pm 3.98 \%)$. Therefore, the lack of an effect of GR blockade on retrieval of text memory might be due to the fact that participants were at ceiling.

One limitation of our studies is that spironolactone and mifepristone act not only on MR and GR, but also on other steroid receptors. Especially mifepristone is known as an anti-progesterone agent (Cadepond et al, 1997). This is particularly important, as recent results show that progesterone acts in concert with glucocorticoids on memory formation (Felmingham et al, 2012). Our study is further limited in that we only examined young men in order to prevent potential side effects of mifepristone in young women. Future studies should investigate whether the results can be generalized to women (at least for spironolactone), and perhaps also to older people.

To summarize, the present study shows that blockade of MRs impairs free recall, in particular of emotional material in young healthy men. In contrast, blockade of GRs enhanced free recall in young healthy men. In combination with previous studies that indicate an impairing effect of elevated cortisol levels on retrieval, the present findings suggest that the previously proposed inverted- $U$-shaped function between memory retrieval and circulating cortisol levels (Lupien and Lepage, 2001; Domes et al, 2005) arises from a differential activation of MRs and GRs and the opposite function each receptor subserves during memory retrieval. The mere proportion of activated MRs and GRs explains the effects of different cortisol levels on memory retrieval well: Memory retrieval is optimal when at intermediate cortisol levels, due to their higher affinity for cortisol, MRs are predominantly occupied but concurrent GR activation is low. Impairment of memory retrieval occurs if MRs are not occupied as a result of very low cortisol levels or if GRs are predominantly activated as a result of very high cortisol levels, indicating the lower and the upper levels of the inverted- $U$-shaped function, respectively.

\section{ACKNOWLEDGEMENTS}

This research is supported by grants from the Deutsche Forschungsgemeinschaft DFG (RI 1894/2-1 and SFB 654) and from the Swiss National Science Foundation (PBZH1118850 , to UR). We thank Timo Guenther, Jonas Klameth, Christian Krumrey, and Boris von Niessen for help with data acquisition.

\section{DISCLOSURE}

The authors declare no conflict of interest.

\section{REFERENCES}

Belanoff JK, Flores BH, Kalezhan M, Sund B, Schatzberg AF (2001). Rapid reversal of psychotic depression using mifepristone. J Clin Pharmacol 21: 516-521.

Born J, Steinbach D, Dodt C, Fehm HL (1997). Blocking of central nervous mineralocorticoid receptors counteracts inhibition of pituitary-adrenal activity in human sleep. J Clin Endocrinol Metab 82: 1106-1110. 
Bradley MM, Greenwald MK, Petry MC, Lang PJ (1992). Remembering pictures: pleasure and arousal in memory. J Exp Psychol Learn Mem Cogn 18: 379-390.

Brickenkamp R, Zillmer E (1998). The d2 Test of Attention. Hogrefe \& Huber: Seattle, Washington.

Buchanan TW (2007). Retrieval of emotional memories. Psychol Bull 133: 761-779.

Buchanan TW, Tranel D (2008). Stress and emotional memory retrieval: effects of sex and cortisol response. Neurobiol Learn Mem 89: 134-141.

Buchanan TW, Tranel D, Adolphs R (2006). Impaired memory retrieval correlates with individual differences in cortisol response but not autonomic response. Learn Mem 13: 382-387.

Cadepond F, Ulmann A, Baulieu EE (1997). RU486 (mifepristone): mechanisms of action and clinical uses. Annu Rev Med 48: 129-156.

Cornelisse S, Joels M, Smeets T (2011). A randomized trial on mineralocorticoid receptor blockade in men: effects on stress responses, selective attention, and memory. Neuropsychopharmacology 36: 2720-2728.

de Kloet ER, Vreugdenhil E, Oitzl MS, Joels M (1998). Brain corticosteroid receptor balance in health and disease. Endocr Rev 19: 269-301.

de Kloet ER, Van Acker SA, Sibug RM, Oitzl MS, Meijer OC, Rahmouni $\mathrm{K}$ et al (2000). Brain mineralocorticoid receptors and centrally regulated functions. Kidney Int 57: 1329-1336.

de Quervain DJ, Roozendaal B, McGaugh JL (1998). Stress and glucocorticoids impair retrieval of long-term spatial memory. Nature 394: 787-790.

de Quervain DJ, Roozendaal B, Nitsch RM, McGaugh JL, Hock C (2000). Acute cortisone administration impairs retrieval of long-term declarative memory in humans. Nat Neurosci 3: 313-314.

Deuschle M, Weber B, Colla M, Muller M, Kniest A, Heuser I (1998). Mineralocorticoid receptor also modulates basal activity of hypothalamus-pituitary-adrenocortical system in humans. Neuroendocrinology 68: 355-360.

Dodt C, Kern W, Fehm HL, Born J (1993). Antimineralocorticoid canrenoate enhances secretory activity of the hypothalamuspituitary-adrenocortical (HPA). axis in humans. Neuroendocrinology 58: 570-574.

Domes G, Rothfischer J, Reichwald U, Hautzinger M (2005). Inverted-U function between salivary cortisol and retrieval of verbal memory after hydrocortisone treatment. Behav Neurosci 119: 512-517.

Douma BR, Korte SM, Buwalda B, la Fleur SE, Bohus B, Luiten PG (1998). Repeated blockade of mineralocorticoid receptors, but not of glucocorticoid receptors impairs food rewarded spatial learning. Psychoneuroendocrinology 23: 33-44.

Dorey R, Pierard C, Chauveau F, David V, Beracochea D (2012). Stress-induced memory retrieval impairments: different timecourse involvement of corticosterone and glucocorticoid receptors in dorsal and ventral hippocampus. Neuropsychopharmacology 37: 2870-2880.

Dorey R, Pierard C, Shinkaruk S, Tronche C, Chauveau F, Baudonnat $\mathrm{M}$ et al (2011). Membrane mineralocorticoid but not glucocorticoid receptors of the dorsal hippocampus mediate the rapid effects of corticosterone on memory retrieval. Neuropsychopharmacology 36: 2639-2649.

Durmer JS, Dinges DF (2005). Neurocognitive consequences of sleep deprivation. Semin Neurol 25: 117-129.

Felmingham KL, Fong WC, Bryant RA (2012). The impact of progesterone on memory consolidation of threatening images in women. Psychoneuroendocrinology 37: 1896-1900.

Ferguson D, Sapolsky R (2008). Overexpression of mineralocorticoid and transdominant glucocorticoid receptor blocks the impairing effects of glucocorticoids on memory. Hippocampus 18: $1103-1111$.
Görtelmeyer R (1985). On the development of a standardized sleep inventory for the assessment of sleep. In Methods of Sleep Research. (Kubicki S, Herman WM eds). (Gustav-Fischer Verlag: Stuttgart, Germany, pp 93-98.

Groch S, Wilhelm I, Diekelmann S, Sayk F, Gais S, Born J (2011). Contribution of norepinephrine to emotional memory consolidation during sleep. Psychoneuroendocrinology 36: $1342-1350$

Heuser I, Deuschle M, Weber A, Kniest A, Ziegler C, Weber B et al (2000). The role of mineralocorticoid receptors in the circadian activity of the human hypothalamus-pituitary-adrenal system: effect of age. Neurobiol Aging 21: 585-589.

Holdstock JS, Mayes AR, Gong QY, Roberts N, Kapur N (2005). Item recognition is less impaired than recall and associative recognition in a patient with selective hippocampal damage. Hippocampus 15: 203-215.

Joels M, de Kloet ER (1994). Mineralocorticoid and glucocorticoid receptors in the brain. Implications for ion permeability and transmitter systems. Prog Neurobiol 43: 1-36.

Kalman BA, Spencer RL (2002). Rapid corticosteroid-dependent regulation of mineralocorticoid receptor protein expression in rat brain. Endocrinology 143: 4184-4195.

Killgore WD (2010). Effects of sleep deprivation on cognition. Prog Brain Res 185: 105-129.

Krug R, Born J, Rasch B (2006). A 3-day estrogen treatment improves prefrontal cortex-dependent cognitive function in postmenopausal women. Psychoneuroendocrinology 31: 965-975.

Kuhlmann S, Piel M, Wolf OT (2005). Impaired memory retrieval after psychosocial stress in healthy young men. J Neurosci 25: 2977-2982.

Lai M, Horsburgh K, Bae SE, Carter RN, Stenvers DJ, Fowler JH et al (2007). Forebrain mineralocorticoid receptor overexpression enhances memory, reduces anxiety and attenuates neuronal loss in cerebral ischaemia. Eur J Neurosci 25: 1832-1842.

Lang PJ, Bradley MM, Cuthbert BN (2005). International Affective Picture System (IAPS): affective ratings of pictures and instruction manual. University of Florida: Gainesville, Florida, Technical Report A-6.

Li TC, Dockery P, Thomas P, Rogers AW, Lenton EA, Cooke ID (1988). The effects of progesterone receptor blockade in the luteal phase of normal fertile women. Fertil Steril 50: 732-742.

Lupien SJ, Lepage M (2001). Stress, memory, and the hippocampus: can't live with it, can't live without it. Behav Brain Res 127: 137-158.

Lupien SJ, Gillin CJ, Hauger RL (1999). Working memory is more sensitive than declarative memory to the acute effects of corticosteroids: a dose-response study in humans. Behav Neurosci 113: 420-430.

Lupien SJ, Wilkinson CW, Briere S, Menard C, Ng Ying Kin NM, Nair NP (2002). The modulatory effects of corticosteroids on cognition: studies in young human populations. Psychoneuroendocrinology 27: 401-416.

Marin MF, Hupbach A, Maheu FS, Nader K, Lupien SJ (2011). Metyrapone administration reduces the strength of an emotional memory trace in a long-lasting manner. J Clin Endocrinol Metab 96: E1221-E1227.

Mayes AR, Holdstock JS, Isaac CL, Hunkin NM, Roberts N (2002). Relative sparing of item recognition memory in a patient with adult-onset damage limited to the hippocampus. Hippocampus 12: $325-340$.

Oitzl MS, de Kloet ER (1992). Selective corticosteroid antagonists modulate specific aspects of spatial orientation learning. Behav Neurosci 106: 62-71.

Oitzl MS, Fluttert M, Sutanto W, de Kloet ER (1998). Continuous blockade of brain glucocorticoid receptors facilitates spatial learning and memory in rats. Eur J Neurosci 10: 3759-3766. 
Otte C, Moritz S, Yassouridis A, Koop M, Madrischewski AM, Wiedemann $\mathrm{K}$ et al (2007). Blockade of the mineralocorticoid receptor in healthy men: effects on experimentally induced panic symptoms, stress hormones, and cognition. Neuropsychopharmacology 32: 232-238.

Otte C, Hinkelmann K, Moritz S, Yassouridis A, Jahn H, Wiedemann K et al (2010). Modulation of the mineralocorticoid receptor as add-on treatment in depression: a randomized, double-blind, placebo-controlled proof-of-concept study. J Psychiatr Res 44: 339-346.

Patel PD, Lopez JF, Lyons DM, Burke S, Wallace M, Schatzberg AF (2000). Glucocorticoid and mineralocorticoid receptor mRNA expression in squirrel monkey brain. J Psychiatr Res 34: 383-392.

Perlman WR, Webster MJ, Herman MM, Kleinman JE, Weickert CS (2007). Age-related differences in glucocorticoid receptor mRNA levels in the human brain. Neurobiol Aging 28: 447-458.

Pryce CR (2008). Postnatal ontogeny of expression of the corticosteroid receptor genes in mammalian brains: interspecies and intra-species differences. Brain Res Rev 57: 596-605.

Rechtschaffen A, Kales A (1968). A manual of standardized terminology, techniques and scoring system for sleep stages of human subjects (NIH Publication No. 204). National Institutes of Health: Bethesda, MD.

Reul J, de Kloet ER (1985). Two receptor systems for corticosterone in the rat brain: microdistribution and differential occupation. Endocrinology 117: 2505-2511.

Rimmele U, Meier F, Lange T, Born J (2010). Suppressing the morning rise in cortisol impairs free recall. Learn Mem 17: 186-190.

Schaff EA, Fielding SL, Westhoff C, Ellertson C, Eisinger SH, Stadalius LS et al (2000). Vaginal misoprostol administered 1, 2, or 3 days after mifepristone for early medical abortion: a randomized trial. JAMA 284: 1948-1953.

Schuerer-Necker E (1994). Gedächtnis und Emotion: Zum Einfluss von Emotionen auf das Behalten von Texten [Memory and emotion: on the influence of emotions on text retention]. Psychologie Verlags Union: Munich.

Schwabe L, Schachinger H, de Kloet ER, Oitzl MS (2010). Corticosteroids operate as a switch between memory systems. J Cogn Neurosci 22: 1362-1372.

Spitz IM, Bardin CW (1993). Mifepristone (RU 486)-a modulator of progestin and glucocorticoid action. $N$ Engl J Med 329: 404-412.

Sternberg S (1966). High-speed scanning in human memory. Science 153: 652-654.

Swahn ML, Bygdeman M, Cekan S, Xing S, Masironi B, Johannisson E (1990). The effect of RU 486 administered during the early luteal phase on bleeding pattern, hormonal parameters and endometrium. Hum Reprod 5: 402-408.

Swain SA, Polkey CE, Bullock P, Morris RG (1998). Recognition memory and memory for order in script-based stories following frontal lobe excisions. Cortex 34: 25-45.

Wagner U, Gais S, Born J (2001). Emotional memory formation is enhanced across sleep intervals with high amounts of rapid eye movement sleep. Learn Mem 8: 112-119.
Wagner U, Degirmenci M, Drosopoulos S, Perras B, Born J (2005). Effects of cortisol suppression on sleep-associated consolidation of neutral and emotional memory. Biol Psychiatry 58: 885-893.

Watson D, Clark LA, Tellegen A (1988). Development and validation of brief measures of positive and negative affect: the PANAS scales. J Pers Soc Psychol 54: 1063-1070.

Watson S, Gallagher P, Porter RJ, Smith MS, Herron LJ, Bulmer S et al (2012). A randomized trial to examine the effect of mifepristone on neuropsychological performance and mood in patients with bipolar depression. Biol Psychiatry 72: 943-949.

Wechsler D (1981). Wechsler Adult Intelligence Scale-Revised. Harcourt Brace Jovanovich: New York.

Wellhoener P, Born J, Fehm HL, Dodt C (2004). Elevated resting and exercise-induced cortisol levels after mineralocorticoid receptor blockade with canrenoate in healthy humans. J Clin Endocrinol Metab 89: 5048-5052.

Wiedemann K, Lauer CJ, Hirschmann M, Knaudt K, Holsboer F (1998). Sleep-endocrine effects of mifepristone and megestrol acetate in healthy men. Am J Physiol Endocrinol Metab 274: E139-E145.

Wiedemann K, Lauer C, Loycke A, Pollmacher T, Durst P, Macher JP et al (1992). Antiglucocorticoid treatment disrupts endocrine cycle and nocturnal sleep pattern. Eur Arch Psychiatry Clin Neurosci 241: 372-375.

Wilhelm I, Wagner U, Born J (2011). Opposite effects of cortisol on consolidation of temporal sequence memory during waking and sleep. J Cogn Neurosci 23: 3703-3712.

Wolf OT (2009). Stress and memory in humans: twelve years of progress? Brain Res 1293: 142-154.

Yau JL, Noble J, Seckl JR (1999). Continuous blockade of brain mineralocorticoid receptors impairs spatial learning in rats. Neurosci Lett 277: 45-48.

Yau JL, Noble J, Seckl JR (2011). 11beta-hydroxysteroid dehydrogenase type 1 deficiency prevents memory deficits with aging by switching from glucocorticoid receptor to mineralocorticoid receptor-mediated cognitive control. J Neurosci 31: 4188-4193.

Young EA, Lopez JF, Murphy-Weinberg V, Watson SJ, Akil H (1998). The role of mineralocorticoid receptors in hypothalamicpituitary-adrenal axis regulation in humans. J Clin Endocrinol Metab 83: 3339-3345.

Young AH, Gallagher P, Watson S, Del-Estal D, Owen BM, Ferrier N (2004). Improvements in neurocognitive function and mood following adjunctive treatment with mifepristone (RU-486) in bipolar disorder. Neuropsychopharmacology 29: $1538-1545$.

Zhou M, Kindt M, Joels M, Krugers HJ (2011). Blocking mineralocorticoid receptors prior to retrieval reduces contextual fear memory in mice. PloS One 6: e26220.

This work is licensed under a Creative Commons Attribution-NonCommercial-NoDerivs 3.0 Unported License. To view a copy of this license, visit http:// creativecommons.org/licenses/by-nc-nd/3.0/ 